\title{
Avulsión postraumática de globo ocular
}

\section{Post-traumatic eyeball luxation}

\author{
Fernando Munayco-Guillén ${ }^{1,2 *}$, Pedro Muro-Mansilla ${ }^{1,3}$, Jose Velasco-Stoll ${ }^{1}$, Jose A. Zavala-Loayza ${ }^{1}$ \\ y Ramón R. Cámara-Reyes ${ }^{4}$ \\ ${ }^{1}$ Instituto Nacional de Oftalmología "Dr. Francisco Contreras Campos", Lima; ${ }^{2}$ Universidad de San Martín de Porres, Lima; ${ }^{3}$ Universidad Peruana \\ Cayetano Heredia, Lima; ${ }^{4}$ Sociedad Científica de Estudiantes de Medicina de Ica (SOCEMI), Universidad San Luis Gonzaga de Ica, Ica. Perú
}

\section{Resumen}

La avulsión del globo ocular es una complicación infrecuente de un trauma de alta energía de la cara, órbita o cráneo. Paciente varón de 20 años en estado etílico sufre un accidente de tránsito en una motocicleta. Señala que se golpeó la cara y el ojo derecho. Niega haber perdido la conciencia, pero nota la pérdida súbita de visión en su ojo derecho. Al examen físico se aprecia el globo ocular luxado de la órbita, con desinserción del músculo recto medial e inferior. Presenta una agudeza visual de no percepción de luz. Antecedente de una craneotomía por un traumatismo craneoencefálico hace 4 años. Se le realiza tomografía computarizada que señala la ausencia del globo ocular derecho en la cavidad orbitaria y no hay fractura ni edema cerebral. Se retira el ojo y se confirmó la luxación total del globo ocular, los músculos extraoculares estaban parcialmente seccionados y el nervio óptico, totalmente seccionado. Se describen diversos mecanismos que explican la salida del globo ocular.

Palabras clave: Avulsión postraumática. Enucleación postraumática. Trauma facial. Trauma craneal. Trauma ocular. Luxación ocular.

\section{Abstract}

Eyeball luxation is an uncommon complication of high-impact trauma to the face, orbit, or skull. This is the case of a 20-yearold male who suffered an accident while driving a motorcycle intoxicated. He points out that he hit his face and his right eye. He denies losing consciousness, but he notices sudden loss of vision in the right eye. Physical examination revealed eyeball luxation from the orbit, with disinsertion of the medial and inferior rectus muscle. Visual acuity was no light perception in his right eye. He has a history of craniotomy due to a prior head injury 4 years ago. A computed tomography showed eyeball absence in the right orbit, without fracture or cerebral edema. In the operating room, an eyeball luxation was confirmed, with partial section of the extraocular muscles and complete avulsion of the optic nerve. We describe several mechanisms that may explain the eyeball luxation.

Key words: Post traumatic luxation. Post traumatic enucleation. Facial trauma. Head trauma. Ocular trauma. Eye luxation. 


\section{Introducción}

El término avulsión significa «extracción por la fuerza». La avulsión postraumática del globo ocular es una condición clínica muy infrecuente. Está asociada a traumatismos faciales y craneoencefálicos ${ }^{1}$.

Se clasifica en: avulsión incompleta, cuando afecta solo el nervio óptico, y avulsión completa, cuando compromete el nervio y los músculos extraoculares ${ }^{2,3}$.

Según Morris, existen tres posibles mecanismos de acción: a) un objeto que entra en la órbita y ejerce la función de cuña o palanca al globo ocular; b) fracturas del hueso de la órbita que desplazan el globo, y c) la fuerza de desaceleración. Existen otros mecanismos como: estrechamiento de la órbita por fractura, hemorragia orbitaria ${ }^{4}$.

El objetivo es describir un caso de avulsión postraumática del globo ocular posterior a un trauma en el macizo facial y el cráneo y discutir su manejo más apropiado.

\section{Presentación del caso}

\section{Caso clínico}

Se trata de un paciente varón de 20 años, que estando en estado etílico sufre un accidente de tránsito cuando iba como pasajero en la parte posterior de una motocicleta. El paciente señala que se golpeó la cara y el ojo derecho, y que presentó pérdida súbita de visión en este ojo. Niega pérdida de conciencia.

El paciente fue auxiliado y llevado a un hospital general donde fue examinado por un neurocirujano, quien a la clínica y al solicitarle una tomografía computarizada de cráneo y órbitas no evidencia signos de fractura o de edema cerebral y lo cataloga como un paciente neurológicamente estable con un Glasgow de 15/15, por lo que decide enviarlo a nuestro instituto para que se le realice una evaluación oftalmológica. No se realizó resonancia magnética por falta de medios económicos y porque el paciente no contaba con un seguro médico.

El paciente llega a nuestra institución aproximadamente después de 15 horas de pasado el suceso. Al examen ectoscópico se evidencia que el globo ocular derecho se encuentra íntegro, pero fuera de la cavidad orbitaria (Figs. 1 y 2). Se observa que la córnea tiene un edema de una cruz (+), lo cual permite visualizar detalles de la cámara anterior, pupila semidriática que no reacciona a la luz y fondo de ojo no evaluable por presencia de hemovítreo, desinserción total de los músculos recto medial y recto inferior.

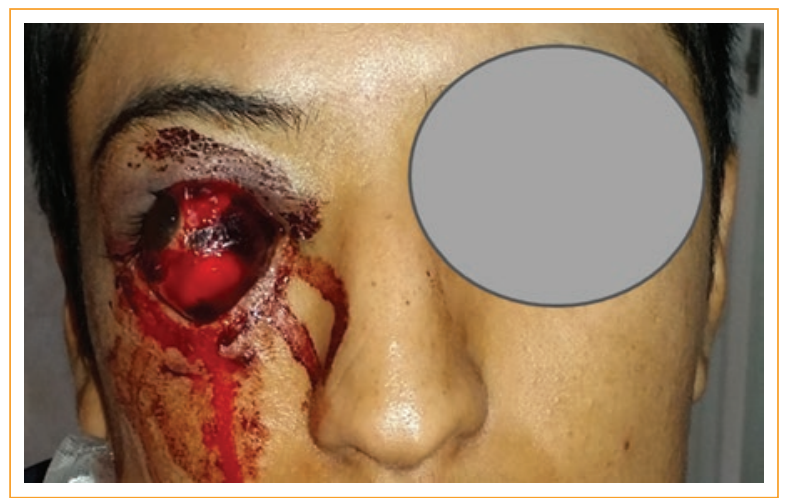

Figura 1. Avulsión postraumática del globo ocular derecho. Nótese la córnea totalmente lateralizada hacia el canto externo.

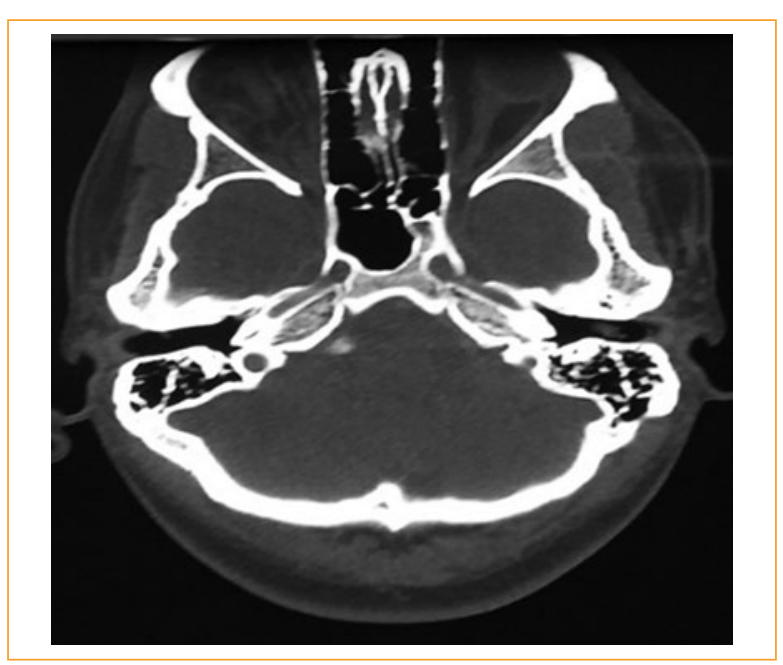

Figura 2. Tomografía que muestra la ausencia del globo ocular derecho en la órbita. No se evidencia signos de fractura ósea en pared orbitaria.

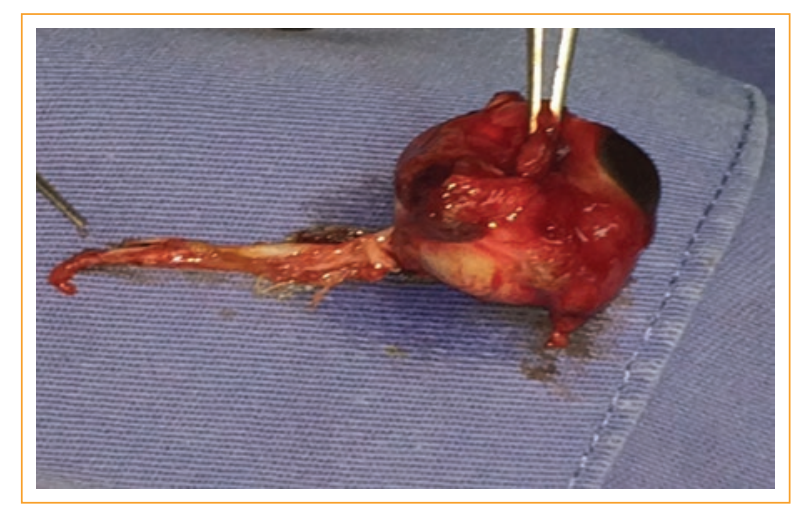

Figura 3. Globo ocular avulsionado con $25 \mathrm{~mm}$ de nervio óptico.

Presentó al ingreso en nuestra institución una agudeza visual en el ojo derecho de no percepción de luz y 20/30 en el izquierdo. Se decide realizar una exploración 


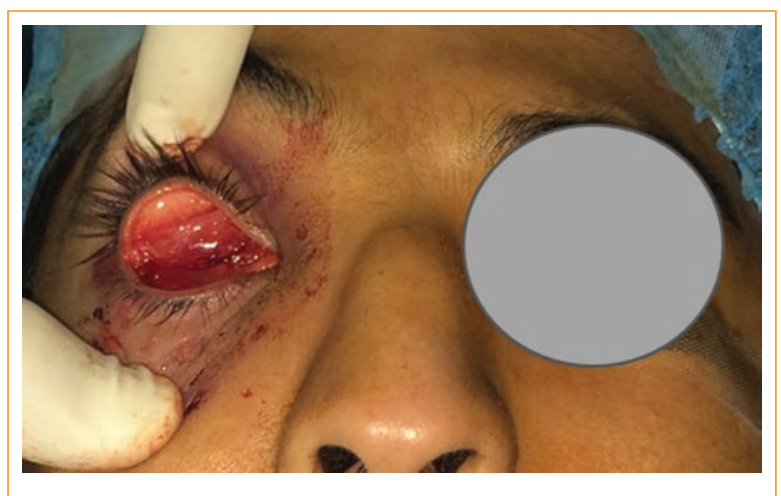

Figura 4. Paciente postenucleación.

quirúrgica para evaluar los daños anatómicos y ver la posibilidad de recolocación del globo ocular en la órbita. Se encontró que el nervio óptico estaba seccionado conjuntamente con las arterias ciliares cortas posteriores y que el globo ocular solo contaba con la circulación colateral de los músculos extraoculares, por lo que se decide completar la enucleación (Figs. 3 y 4). Al evidenciar la sección total del recto medial e inferior y el daño extenso en la superficie ocular, se consideró alta la posibilidad de exposición y extrusión de un implante, por lo que se decidió no colocarlo (Fig. 4).

La muestra fue enviada para su estudio al laboratorio de patología ocular, el cual encontró presencia de fibrina en la cámara anterior, sinequias iridocristalinianas, desprendimiento del cuerpo ciliar, hemorragia coroidea y supracoroidea, hemovítreo denso, necrosis retinal, atrofia óptica y meninges sin nervio óptico.

\section{Discusión}

La avulsión postraumática del globo ocular es una condición clínica muy infrecuente y, por lo general, está relacionada con el trauma de las estructuras maxilofaciales $^{1,2}$. En nuestro caso no hubo fracturas de huesos de la órbita, cráneo, según imágenes tomográficas.

Todo paciente con avulsión del nervio óptico y luxación del globo ocular postrauma debe tener primero un examen clínico neurológico completo que incluya un control tomográfico y de resonancia magnética. Se recomienda iniciar terapia antibiótica de amplio espectro para prevenir infecciones ${ }^{1,3}$.

Dependiendo del estado anatómico y visual del globo ocular, se debe tratar de reposicionar en la órbita por su beneficio estético y psicológico. La reposición del globo ocular depende del estado funcional del ojo, la integridad anatómica del globo ocular, la presencia de infecciones, el estado vascular ocular, la vitalidad de los tejidos oculares, la presencia de dolor ${ }^{4,5}$. El recolocar el globo ocular en la órbita permite evitar al paciente la sensación de mutilación, conseguir un mejor resultado estético. En el caso de los niños favorece y estimula el desarrollo orbitario $0^{6,7}$.

Aunque en algunos casos el globo ocular evolucione después a la ptisis bulbi, se ha visto que, un tiempo después, el paciente muestra más aceptación a someterse a una cirugía de evisceración para mejorar su aspecto estético ${ }^{7}$. Si el daño no involucró totalmente la arteria oftálmica, el globo ocular difícilmente llegará a la ptisis bulbi?.

La literatura señala que, en este tipo de traumas, el orden de avulsión de los músculos extraoculares según la frecuencia es: recto medio, recto inferior, recto lateral y oblicuos ${ }^{5,6,8}$. En nuestro paciente, este dato es concordante.

Teniendo en cuenta los mecanismos de acción, nuestro paciente refiere que no se le introdujo ningún objeto extraño en su órbita, por lo que presumimos que uno de los principales mecanismos fue la desaceleración, la cual es descrita por Song y Carter ${ }^{6}$.

\section{Conclusiones}

La avulsión postraumática del globo ocular es una condición clínica muy infrecuente, pero está muy relacionada con el trauma de estructuras faciales y orbitarias.

Se debe evaluar a estos pacientes de manera integral, desde la perspectiva de cada especialidad: neurología, oftalmología, cirugía de cabeza y cuello.

Dependiendo del estado anatómico y visual del globo ocular, se debe tratar de reposicionar en la órbita por su beneficio estético y psicológico.

\section{Conflicto de intereses}

Los autores no tienen ningún conflicto de intereses.

\section{Financiamiento}

El presente artículo fue autofinanciado.

\section{Responsabilidades éticas}

Protección de personas y animales. Los autores declaran que para esta investigación no se han realizado experimentos en seres humanos ni en animales. 
Confidencialidad de los datos. Los autores declaran que han seguido los protocolos de su centro de trabajo sobre la publicación de datos de pacientes.

Derecho a la privacidad y consentimiento informado. Los autores han obtenido el consentimiento informado de los pacientes y/o sujetos referidos en el artículo. Este documento obra en poder del autor de correspondencia.

\section{Bibliografía}

1. Gupta R. Traumatic complete evulsion of the globe and optic nerve. Can J Ophthalmol. 2014;49(5):110-2.
2. Pillai S, Mahmood M, Limay S. Complete evulsion of the globe and optic nerve. Br J Ophthalmol. 1987;71:69-72.

3. De Santana Santos T, Vajgel A, Ribeiro CF, de Santana Júnior JR, Andrade Filho ES. Avulsion of globe following maxillofacial trauma. J Craniofac Surg. 2012;23(4):1097-100.

4. Figueiredo-Amaral M, Furtado-Carvalho M, Baptista-Ferreira A, Alves-Mesquita $R$. Traumatic globe luxation associated with orbital fracture in a child: A case report and literature review. J Maxillofac Oral Surg. 2015;4(1):323-30.

5. Kumari E, Chakraborty S, Ray B. Traumatic globe luxation: A case report. Indian J Ophthalmol. 2015;63:682-4.

6. Kelahmetoğlu O, Șimşek T, Beden U. Rarely seen complication of motor vehicle accidents: Bilateral globe avulsión. Turk J Trauma Emerg Surg. 2015;21(4):297-9.

7. Ersan I, Adam M, Oltulu R, Zengin, Okka M. Traumatic luxation of the globe: A 6-year follow-up. Orbit. 2016;35(2):69-71.

8. Tok L, Tok OY, Argun T, Yilmaz O, Alime G, Elif U, et al. Bilateral traumatic globe luxation with optic nerve transection. Case Rep Ophthalmol. 2014:5:429-34. 